\title{
A Novel Liquid Antenna for Wearable Bio-monitoring Applications
}

\author{
$\underline{\text { Anya Traille }}^{1,2}$, Li Yang $^{1}$, Amin Rida $^{1}$ and Manos M. Tentzeris ${ }^{1}$ \\ ${ }^{1}$ GEDC/ECE, Georgia Tech, Atlanta, GA 30332-250, USA, ${ }^{2}$ GTRI/SEAL, Georgia Tech, USA \\ email:atraille@ece.gatech.edu
}

\begin{abstract}
The performance of the most commonly used metal antennas close to the human body is one of the limiting factors of the performance of bio-sensors and wireless body area networks (WBAN). Due to the high dielectric and conductivity contrast with respect to most parts of the human body (blood, skin, ...), the range of most of the wireless sensors operating in RF and microwave frequencies is limited to $1-2 \mathrm{~cm}$ when attached to the body. In this paper, we introduce the very novel idea of liquid antennas, that is based on engineering the properties of liquids. This approach allows for the improvement of the range by a factor of 5-10 in a very easy-to-realize way, just modifying the salinity of the aqueous solution of the antenna. A similar methodology can be extended to the development of liquid RF electronics for implantable devices and wearable realtime bio-signal monitoring, since it can potentially lead to very flexible antenna and electronic configurations.
\end{abstract}

Index Terms - Liquid antennas, bio-monitoring, wireless body area networks.

\section{INTRODUCTION}

The explosive growth of the biosensors and health-related wearable monitoring devices has accentuated the need for miniaturized, high-efficiency conformal materials that can operate over a wide range of frequencies, while they can be integrated in wearable and lightweight configurations. One of the major issues for the implementation of Wireless Body Area Networks (WBAN) of these devices is the very limited range of commonly used metal antennas. Due to the high dielectric constant (Table 1) between the metal antenna material (as well as the metal-based circuitry) and the human body tissues consisting mostly of "ionized-water", the nearfield is significantly disturbed, while local reflections due to the dielectric mismatch cause unwanted distortion in the reading image, that may require extensive reconstruction to produce a sufficient quality for accurate analysis. Current forms of bio-monitoring are invasive in that they involve the application of stimulation currents by attaching electrodes directly to the body or skin, posing the risk of harming the immediate and surrounding tissue due to electrolysis at the electrode-tissue interface.

The goal of the liquid antenna is the same. Since the electrical properties of the body vary with tissue type, temperature, water content, organ movement and blood flow, it is possible that the resulting variation of a liquid antenna's radiation pattern with inhomogeneous dielectric properties can be used to communicate information about a person' health condition such as body temperature, tissue damage, tumors, inflammation and breathing. Like other bio-sensing devices, to detect internal bio-activities based on the dielectric and conductive properties of tissue, the microwave signal must be able to penetrate into the tissue posing the need to utilize liquids with a high dielectric constant in order to better focus and direct the electromagnetic energy.

The idea of a liquid antenna was explored as early as 1970 where Ting and King [1] proved theoretically that dielectric filled tubes become resonant at specific frequencies. Ida [2] demonstrated the dependence of the efficiency bandwidth on the permittivity of the dielectric surrounding a metal conductor. This dielectric slows the velocity of the electromagnetic energy and leads to physically smaller than the purely metallic thin-wire counterparts for the same frequency. In this paper, we propose for the first time the development of liquid antennas, based on aquous salt

TABLE I [3]

DiELECTRIC PROPERTIES @ 1.5-2.0GHz

\begin{tabular}{|c|l|}
\hline Metal & $\varepsilon_{r}=1$ \\
\hline Blood & $\varepsilon_{r}=58$ \\
\hline Skin & $\varepsilon_{r}=37.5$ \\
\hline
\end{tabular}

solutions, operating in microwave frequencies (around $1.7 \mathrm{GHz}$ ) with significantly improved range and efficiency characteristics, as well as a much simpler and flexible fabrication mechanism. Encapsulating the proposed liquid antennas in flexible plastic containers makes them quite easily wearable or implantable in the proximity of human organs or biomonitoring devices (e.g pacemakers) in order to enable short-range wireless communication/control. In addition, corrosion resistance is another advantage of the ionic-liquid, glass/plastic-enclosed antennas, while the easy elimination of air gaps permits shape manipulation and an improved electromagnetic coupling between the probe and the probed dielectric. Since the dielectric property and the conductivity of these solutions are a function of the salinity level as well as of the type of the dissolved salt, these antennas can be easily reconfigured for different areas of mounting/observation. 


\section{ELECTRICAL PROPERTIES OF WATER SOLUTIONS}

\section{A. Electrical properties of water}

A Liquid water is a dielectric and a conductor that self ionizes $\left(\mathrm{H}_{2} \mathrm{O} \leftrightarrow \mathrm{H}^{+}+\mathrm{OH}^{-}\right)$. As a dielectric, the finite charge displacement (rotation) of the polar molecules occurring under an electric field is referred to as Maxwell Displacement current. The electrical conduction (collective translational charge movement) occurring in water is not very well understood due to the very complex structural properties of water having to do with the transfer of hydrated water ions (hydronium $\mathrm{H}_{3} \mathrm{O}$, and hydroxide $\mathrm{OH}^{-}$). between various water clusters or chains held together by hydrogen bonds [4].

Adding salt to water is believed to break the structure of water clusters, freeing molecules which in turn have a lower relaxation time, thus causing an overall depolarizing effect in the bulk solution. As the salt concentration increases more, the salt ions reduce the amount of free molecules, eventually causing the relaxation time to decrease again. The overall effect is that adding salt increases the loss due to the drift of ions (conductive loss) and decreases the dielectric constant of water.

When the water (or electrolyte) is biased with electrodes of opposite polarity, the negative electrode attracts the hydrogen atoms while the positive electrode attracts the oxygen atoms. This accelerates the ion transport mechanisms and results in the electrolysis process at the solid/solution interface where $\mathrm{O}_{2}$ is produced at the anode and $\mathrm{H}_{2}$ is produced at the cathode. These processes occur at a potential limit that depends on the electrode materials [5-11]

\section{B. Feeding Process}

At low frequencies, within a particular potential range, the method of charge injection can be a non-faradic process that involves an increase or decrease in water ion concentration at the liquid-electrode (adsorption and desorption) and charge polarization (electrical double layer capacitance) at the liquidelectrode interface but no charge transfer between the liquid and electrode. This is undesirable since the electrical double layer blocks the electric field from the bulk solution and prevents the electric field from coupling to the bulk liquid which is to serve as the conducting antenna element. At higher frequencies, a reversible faradic process may occurs via charge transfer at the interface (oxide formation and reduction) in addition adsorption and desorption of water ions. Since the polarity alteration of the electrodes must cycle rapidly at some resonant frequency, a reversible electrode process is necessary whereas, in the case of an irreversible process, the alternating potential would result in the accumulation of solids or gasses onto the electrode surface over time [5-11].
It is desirable to use electrode materials which are noncorrosive, chemically stable, biocompatible and non-toxic so that they will not induce an immune response on the skin or tissue. Additional material-specific characteristics relate to surface morphology and microstructure, extrinsic exchange density, and adsorption behavior (which can be controlled using coatings). Examples of such materials are platinum, gold, and titanium nitride.

\section{Antenna Liquid Container}

The liquid must be encapsulated in a flexible, noncorrosive, bio-compatible, non-toxic container. Kynar PVDF is a tough, flexible, heat and chemical resistant plastic with an $\varepsilon_{\mathrm{r}}$ of around 8.4. It is available in two grades, the Homopolymer Kynar, which is the semi rigid grade, and the Co-polymer Kynar, which is the flexible grade.

\section{BENCHMARKING STRUCTURE SiMULATIONS}

To characterize the performance of a liquid antenna, we performed extensive simulations for the structure shown in Fig.1. The bracelet-type liquid antenna consisting of a $2.8 \mathrm{M}$ aqueous salt solution $\left(\varepsilon_{\mathrm{r}}=38\right)$ inside a very thin plastic tubing container having a tube radius of $2.5 \mathrm{~mm}$. The bracelet was excited using two electrodes on both sides of the slot) and mounted on various positions of an inhomogeneous multilayer cylinder emulating the human body. This cylinder with the length of $250 \mathrm{~mm}$ (similar to the lower arm) was composed by two materials: for the inner cylinder with radius $r=24 \mathrm{~mm}$, the material was blood with dielectric constant 58 and conductivity $1.2 \mathrm{~S} / \mathrm{m}$, while for the outer cylindrical ring the material was skin with dielectric constant 37.5 and conductivity $0.6 \mathrm{~S} / \mathrm{m}$. The simulation was performed at the central frequency of $1.7 \mathrm{GHz}$.

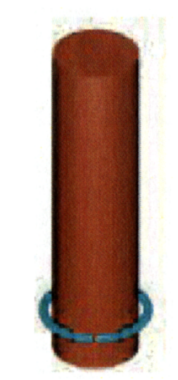

Fig. 1. Wearable liquid antenna bracelet around the lower-arm phantom

Numerous TLM (time-domain) simulations were performed for this structure and the comparative results between a metal (copper) antenna of the same physical size mounted at the same position ( $30 \mathrm{~mm}$ from the edge) with liquid antenna are shown in Figs.2(a),(b). It can be clearly seen that the liquid 
antenna has a maximum to the direction transverse to the arm with efficiency of $59 \%$ and a directivity of $5.64 \mathrm{dBi}$. On the opposite, the metal antenna has the maximum of its radiation pattern along the direction of the arm, thus minimizing its communication range away from the body. The simulated directivity was $3.36 \mathrm{dBi}$ and the efficiency was $26 \%$. Comparison of the range to the direction that is transverse to the body, and it is usually the most critical dimension for the communication of bracelet-mounted wearable biosensors demonstrated that the liquid antenna led to an improvement by a factor of 12 .
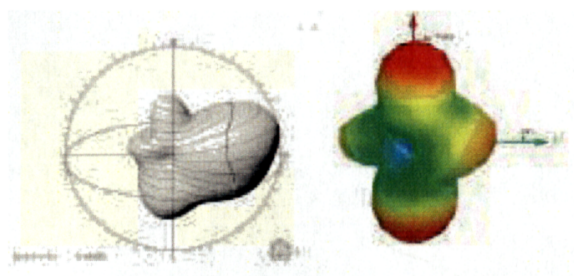

Fig. 2. Radiation patterns for wearable bracelet: (a) liquid antenna, (b) copper antenna arm phantom

Changing the position of the bracelet on the lower-arm phantom, can easily tilt the radiation pattern further improving the directivity and the range of reach. As the bracelet is close to the edge of the arm, the radiation pattern slightly tilts vertically, but still maintains the main pattern characteristics, having the maximum to the direction of the slot. as shown in Figs.3(a),(b). The directivity is $5.86 \mathrm{dBi}$ and $6.44 \mathrm{dBi}$ and the efficiency $61 \%$ and $63 \%$, respectively, for the position of the liquid antenna at $60 \mathrm{~mm}$ from the bottom edge and at $125 \mathrm{~mm}$ from the bottom edge (middle of the arm).

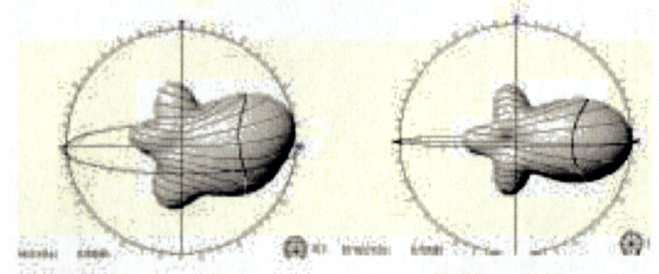

Fig. 3. Radiation patterns for wearable bracelet liquid antenna: (a) $60 \mathrm{~mm}$ from bottom, (b) middle (125 $\mathrm{mm}$ from bottom)

One of the most important features of the proposed novel antenna is its very easy re-configurability by simply changing the salinity levels (or modifying the diluted salt). Timedomain simulations for two different values of salinity $(2.853$ $\mathrm{mol} /$ liter and $4.643 \mathrm{~mol} /$ Liter), shown in Figs. 4(a),(b) demonstrate that as the salinity increases the pattern gets more isotropic, but maintains the radiation characteristics with a peak to the direction that's transverse to the arm. For (a) the dielectric constant was 38 with a conductivity value of 12.4 $\mathrm{S} / \mathrm{m}$ leading to a directivity of $5.64 \mathrm{dBi}$, while for (b) the dielectric constant was 23 with a conductivity of $21 \mathrm{~S} / \mathrm{m}$. It is clear that the dielectric constant and the conductivity values of the saltwater can be very easily modified by changing the salinity. That would allow the easy reconfigurability of this type of structures in order for them to readjust for different types of tissues, such as internal organs, skin and blood. In addition, that feature would enable the easy re-adjustment and operability of this device in different ambient conditions (desert, polar, space environments) when utilized in wearable applications.

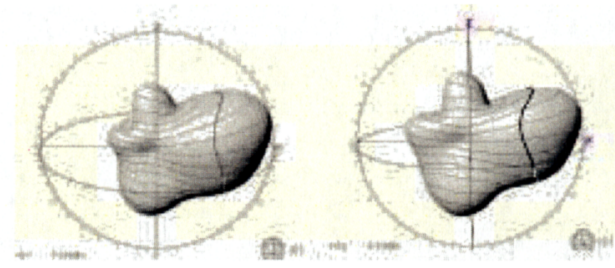

Fig. 4. Radiation patterns for liquid antenna salinities: (a) $2.853 \mathrm{~mol} / \mathrm{Liter}$, (b) $4.634 \mathrm{~mol} / \mathrm{Liter}$

As a proof of concept, we plan to realize a real-size prototype of a wearable bracelet and characterize its performance at $1.7 \mathrm{GHz}$ utilizing numerous possible excitation mechanisms. Comparison results between simulations and measurements will be presented at the conference.

\section{CONCLUSIONS}

We have demonstrated for the first time in RF frequencies the capabilities of the novel concept of liquid antennas for wearable or implantable bio-monitoring applications. Although conventional metal antennas suffer from low range and oxidation problems when in proximity to the human body, the proposed topology features an improved range by up to an order of magnitude. In addition, it is very conformal allowing for an easy wearable WBAN implementation, as well as it can be very easily reconfigured by adjusting the salinity of the salt solutions in order for it to operate in different ambient conditions. The proposed work could set the foundation for the implementation of truly wireless biosensor networks with small-size, lightweight conformal nodes for wireless health monitoring in pharmaceuticals, hospital, ambulance and home-based patient care, as well as in space/underwater missions.

\section{ACKNOWLEDGEMENT}

The authors wish to acknowledge the NSF CAREER ECS9984761, the NSF ECS-0313951, the Georgia Electronic Design Center (GEDC). Also, the authors would like to acknowledge the support of GTRI-IRAD Award. 


\section{REFERENCES}

[1] C. Y. Ting, R. King, "The dielectric-filled tubular monopole," IEEE Transactions on Antennas and Propagation, vol. 18, no. 5, pp. 604-610, Sep. 1970.

[2] I. Ida, J. Sato, T. Sekizawa, H. Yoshimura, K. Ito, "Dependence of the efficiency-bandwidth product on electrical volume of small dielectric loaded antennas", IEEE Transactions on Antennas and Propagation, vol. 50, no. 6, pp. 821-826, Jun. 2002.

[3] D. Andreuccetti, R. Fossi, C. Petrucci. (1997-2007). An internet resources for the calculation of dielectric properties of body tissue in the frequency range $10 \mathrm{~Hz}-100 \mathrm{GHz}$. [http://niremf.ifac.cnr.it/tissprop/].

[4] A. Sadkowski, "On the ideal polarisability of electrodes displaying cpetype capacitance dispersion," Journal of Electroanalytical Chemistry, vol. 481, no. 2, pp. 222-226, Feb. 2000.

[5] S. C. Bera, S. Chattopadhyay, B. Chakraborty, "An experimental analysis of the non-linear behavior of a bio-electrode polarisation impedance with excitation frequency," Measurement, vol. 35, no. 4, pp. 3630-370, Jun. 2004.

[6] A. R. A Rahmanm, D.T. Price, S. Bhansali, "Effect of electrode geometry on the impedance evaluation of tissue and cell culture," Sensors \& Actuators: B. Chemical, vol. 127, no. 1, pp. 89-96, Oct. 2007.
[7] C. Gabriel, S. Gabriel, E. H. Grant, B. S. J. Halstead, D. Michael, P. Mingos, "Dielectric parameters relevant to microwave dielectric heating," Chemical Society Reviews, vol. 27, pp. 213-223, Feb. 1998.

[8] J. J. Whalen, J. Young, J. D. Weiland, P. C. Searson, "Electrochemical Characterization of Charge Injection at Electrodeposited Platinum Electrodes in Phosphate buffered Saline," Journal of the Electrochemical Society, vol. 153, no. 12, pp. C834-C839, Dec. 2006.

[9] H. Zhou, M. A. Preston, R.D. Tilton, L. R. White, "Calculation of the electric polarizability of a charged spherical dielectric particle by the theory of colloidal electrokinetics," Journal of Colloid and Interface Science, vol. 285, pp. 845-856, May. 2005.

[10] R. Somaraju, J. Trumpf, "Frequency, Temperature and Salinity Variation of the Permittivity of Seawater," IEEE Transactions on Antennas and Propagation, vol. 54, no 11, pp. 3441-3448, Nov. 2000.

[11] P.Bruesch, C.Thomas, "The electric double-layer at a metal electrode in pure water," Journal of Applied Physics, vol. 95, no 5, pp. 2846-2856, Mar.2001. 